\title{
RSC Advances
}

Check for updates

Cite this: RSC Adv., 2020, 10, 15293

\section{A rapid and ultrasensitive colorimetric biosensor based on aptamer functionalized Au nanoparticles for detection of saxitoxin}

\author{
Le Qiang, (D) a Yu Zhang, ${ }^{\star a}$ Xin Guo, ${ }^{a}$ Yakun Gao, ${ }^{a}$ Yingkuan Han, ${ }^{\text {ab }}$ Jun Sun (D) \\ and Lin Han (D) *a
}

\begin{abstract}
Saxitoxin (STX) is one of the most important marine toxins which affects the safety of domestic water. Rapid, sensitive and selective recognition of STX is crucial in environment monitoring. Here, we demonstrate a facile and ultrasensitive colorimetric sensor based on gold nanoparticles (Au NPs) and aptamer (Au NPs-aptamer biosensor) for specific and quantitative detection of STX. The aptamer reacts specifically with STX, resulting in the aggregation of Au NPs and the color change of the Au NP solution. The lowest detection concentration of the colorimetric sensor is $10 \mathrm{fM}\left(3 \mathrm{fg} \mathrm{mL}^{-1}\right)$, and a good linear relationship $\left(R^{2}=0.9852\right)$ between the absorbance ratio and STX concentrations (10 fM to $\left.0.1 \mu \mathrm{M}\right)$ indicates that our Au NPs-aptamer biosensor can be used for quantitative sensing of STX. The detection time of STX is 30 minutes, and the sensor is successfully applied in the specific detection of STX in seawater. The Au NPaptamer biosensor shows great potential in practical applications to monitor environmental pollution, marine aquaculture pollution, and seafood safety.
\end{abstract}

Received 8th February 2020

Accepted 9th April 2020

DOI: 10.1039/d0ra01231a

rsc.li/rsc-advances
The traditional analytical techniques for STX include high performance liquid chromatography (HPLC), ${ }^{8}$ liquid chromatography-tandem mass spectrometry (LC-MS), ${ }^{9}$ and enzyme-linked immunosorbent assay (ELISA). ${ }^{10}$ HPLC and LCMS need sophisticated equipment and complex pre-treatment, which limit the detection to the laboratory. ${ }^{\mathbf{1 1 , 1 2}}$ It involves tedious lab work and non-specificity during ELISA methods, ${ }^{13}$ so it is hard to conduct real-time detection. As a result, to realize real-time detection, it desires new approaches to develop a facile, rapid, and sensitive platform to identify and monitor STX.

$\mathrm{Au}$ NPs have been employed as colorimetric sensors due to their excellent optical properties ${ }^{\mathbf{1 4}}$ and their ability to bond biomolecules. ${ }^{15}$ The colorimetric sensor based on Au NPs has the advantages of high sensitivity and simplicity, and is capable of realizing real-time detection without large-scale instruments and equipment. It has been used to detect proteins, ${ }^{\mathbf{1 6 - 1 8}}$ DNAs,${ }^{19-21}$ metal ions, ${ }^{22,23}$ and small molecules. ${ }^{24-27}$ To construct Au NPs based colorimetric sensor, a specific molecule is needed to bind with $\mathrm{Au}$ NPs, which can specifically react with target molecules, and then gets desorbed from Au NPs resulting in the aggregation of Au NPs to induce color change. Here we report an $\mathrm{Au}$ NPs-aptamer based sensor, and the aptamer functionalized on Au NPs specifically reacts with STX, resulting in the aggregation of $\mathrm{Au}$ NPs and the change of the surface plasmon resonance absorption peak, which can be used for the quantitative analysis of STX. Our Au NPs-aptamer based colorimetric sensor achieves the lowest detection concentration of $10 \mathrm{fM}(\sim 3 \mathrm{fg}$ $\left.\mathrm{mL}^{-1}\right)$, and presents a good linear relationship $\left(R^{2}=0.9852\right)$
${ }^{a}$ Institute of Marine Science and Technology, Shandong University, Qingdao 266237, China.E-mail:yuzhang@sdu.edu.cn; hanlin@sdu.edu.cn

${ }^{b}$ School of Microelectronics, Shandong University, Jinan 250010, China

${ }^{c}$ Research Centre for Indian Ocean Ecosystem, Tianjin University of Science and Technology, Tianjin 300457, China 
between the absorbance ratio and STX concentrations (10 fM to $0.1 \mu \mathrm{M})$. The detection time of STX is 30 minutes, and the sensor is successfully applied in the specific detection of STX in seawater. This work opens new applications of colorimetric sensor in marine science and technology.

\section{Materials and methods}

\subsection{Materials}

Sodium chloride $(\mathrm{NaCl})$ was purchased from Solarbio Science and Technology Corporation, Beijing, China. Saxitoxin toxin was purchased from National Research Council-Conseil National de Recherché Canada (NRC-CNRC, Canada). 96 well microplates were polystyrene, flat-bottomed, tissue culture treated (Corning, cat. no. 3595).

\subsection{Au NPs synthesis and characterization}

Gold colloids were prepared by citrate thermal reduction method. ${ }^{28}$ Typically, in the process of thermal reduction, a gold sol was prepared by mixing $1 \mathrm{~mL}$ of $1 \mathrm{wt} \% \mathrm{HAuCl}_{4}$ aqueous solution and $1.5 \mathrm{~mL}$ of $38.8 \mathrm{mM}$ sodium citrate aqueous solution with $90 \mathrm{~mL}$ boiling water. The solution turned purple red within $30 \mathrm{~s}$, and was cooled quickly in the ice bath. ${ }^{29}$ This resulted in a stable dispersion of gold particles with an average diameter of around $14 \mathrm{~nm}$. The microstructure of Au NPs was characterized using a FEI-Nova NanoSEM 450 scanning electron microscope (SEM), and the applied acceleration voltage was 10 $\mathrm{kV}$ with a working distance of $5.0 \mathrm{~mm}$. The high-resolution transmission electron microscope (HRTEM) measurement of Au NPs was performed by a FEI Tecnai G2 F20 electron microscope operated at $200 \mathrm{kV}$. The particle size of Au NPs was calculated by Nano Measurer software.

\subsection{STX aptamer synthesis}

The sequence of STX aptamer was from the literature ${ }^{30}$ and synthesized from Sangon Biotech Corporation, Shanghai, China, and listed in Table 1.

\subsection{General procedure of STX detection}

First, $10 \mu \mathrm{L}$ of $0.36 \mathrm{M} \mathrm{NaCl}$ solution was added into $80 \mu \mathrm{L} \mathrm{Au}$ NPs solution. Then $10 \mu \mathrm{L}$ of $1 \mu \mathrm{M}$ STX aptamer solution was added and incubated with Au NPs for 14 hours. Next, $10 \mu \mathrm{L}$ of tenfold dilutions STX by $\mathrm{f} / 2$ medium $^{31}$ was added into the STX aptamer-Au NPs complex and reacted with aptamer for $30 \mathrm{~min}$. The UV-vis absorption spectra of samples were scanned from

Table 1 Sequences of STX aptamer

\begin{tabular}{llr}
\hline Name & Sequence (from the $5^{\prime}$ to $3^{\prime}$-end) & NT \\
\hline STX aptamer & GGTATTGAGGGTCGCATCCC & 78 \\
& GTGGAAACATGTTCATTG & \\
& GGCGCACTCCGCTTTCTG & \\
& TAGATGGCTCTA & \\
& ACTCTCCTCT
\end{tabular}

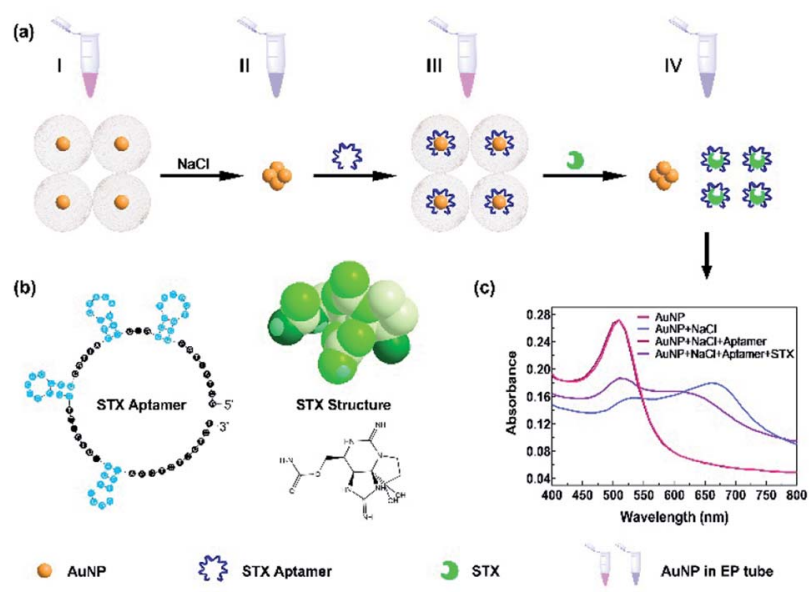

Fig. 1 (a) The detection scheme of colorimetric STX sensor based on Au NPs. (I) Dispersed Au NPs in the initial reaction system; (II) Au NPs aggregated with $\mathrm{NaCl}$ input; (III) Au NPs back to dispersion status after binding with aptamer; (IV) Au NPs aggregated after STX loading. (b) Structure of STX aptamer and STX. (c) UV-vis spectra of Au NPs under different conditions.

400 to $800 \mathrm{~nm}$ using a BioTek microplate reader (Synergy ${ }^{\mathrm{TM}} \mathrm{H} 1$ ) with pure water as the control in 96 well microplates. A working curve was drawn in which $x$ and $y$-coordinates were wavelength and absorption spectra, respectively. The final volume of all samples was $110 \mu \mathrm{L}$. Each detection was performed at least three times in parallel and GraphPad Prism 8 software (https:// www.graphpad.com/scientific-software/prism/) was used for data analysis.

\section{Results and discussion}

\subsection{Colorimetric detection mechanism of STX based on aptamer functionalized Au NPs}

The colorimetric detection mechanism of STX is based on the aggregation of Au NPs functionalised with aptamer as shown in Fig. 1. Dispersed Au NPs aggregate with appropriate $\mathrm{NaCl}$ concentration, and get back to dispersion status after the binding with aptamer. Once STX is loaded, the aptamer reacts with STX and expose Au NPs to get aggregated. The absorbance peaks changes to form different $A_{680} / A_{520}$ ratios with different STX concentrations, which can be used for the quantitative analysis of STX.

\subsection{Characterization of Au NPs}

Here we employed Au NPs which were prepared by citrate thermal reduction method, and the material characteristics were shown in Fig. 2. SEM image of Au NPs is shown in Fig. 2a, and Fig. 2b presents the statistical size of Au NPs is around $14 \mathrm{~nm}$. The charge balance of Au NPs and recovery after aggregation are the most crucial factors for Au NPs based sensor, which are determined by Au NPs material properties and reaction system of sensing assay, including Au NPs size, optical characteristics, and composites in aqueous reaction. HRTEM images in Fig. 2c and d are Au NPs morphology in 

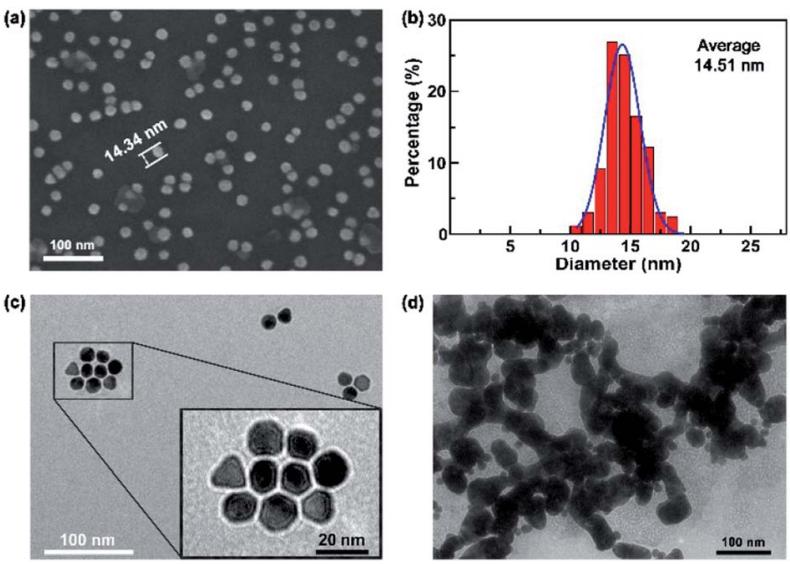

Fig. 2 (a) SEM image of Au NPs. (b) Particle size analysis of Au NPs. HRTEM images of Au NPs (c) in dispersion and (d) in aggregation.

dispersed and aggregated status. Fig. 2c presents good dispersion of Au NPs, most Au NPs are spherical and while few are triangle shape. Dispersed Au NPs are triggered by NaCl or other conditions, and aggregate to form large size particles, as shown in Fig. 2d viewed under HRTEM.

\subsection{Optimization of experimental conditions}

The $\mathrm{NaCl}$ concentration is a critical parameter to induce the aggregation of Au NPs, and detailed study is conducted to investigate the $\mathrm{NaCl}$ concentration threshold for Au NPs aggregation. Here, the used reaction system has Au NPs density of $47.8 \mu \mathrm{g} \mathrm{mL} \mathrm{m}^{-1}$. Fig. 3a shows the representative UV-vis adsorption spectra of Au NPs solution mixed with different concentrations of $\mathrm{NaCl}$ from $0 \mathrm{M}$ to $0.4 \mathrm{M}$, and the $520 \mathrm{~nm}$ peak decreases with increased $\mathrm{NaCl}$ concentration. It shows a sudden
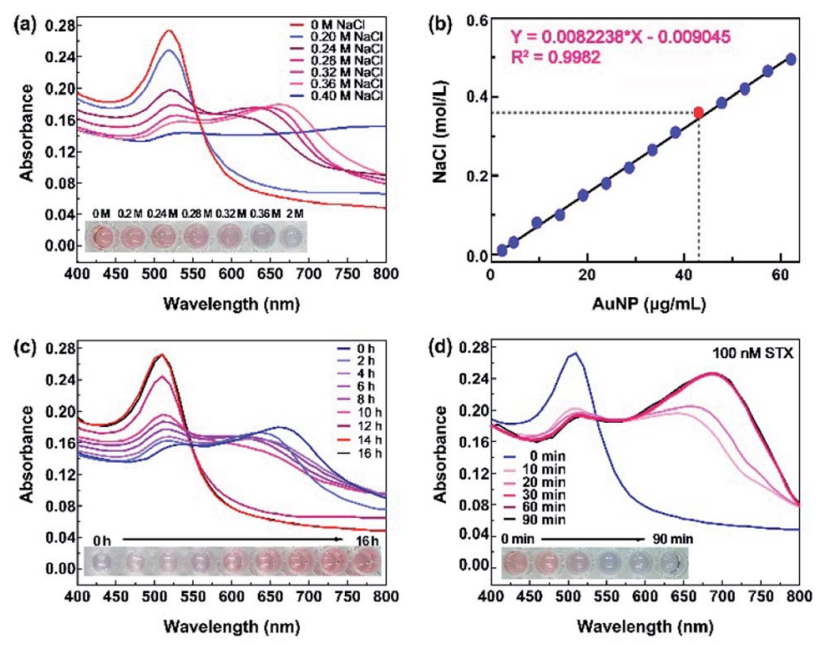

Fig. 3 (a)The alteration of Au NPs aggregation in different $\mathrm{NaCl}$ concentrations including color and absorption spectra. (b) Function of $\mathrm{Au} \mathrm{NPs}$ and concentrations of aggregated $\mathrm{NaCl}$. The red dot is the threshold we chose. (c) The alteration of color and absorption spectra with STX aptamer incubation time. (d) The absorption spectra and alteration of color with reaction time after loading STX samples.
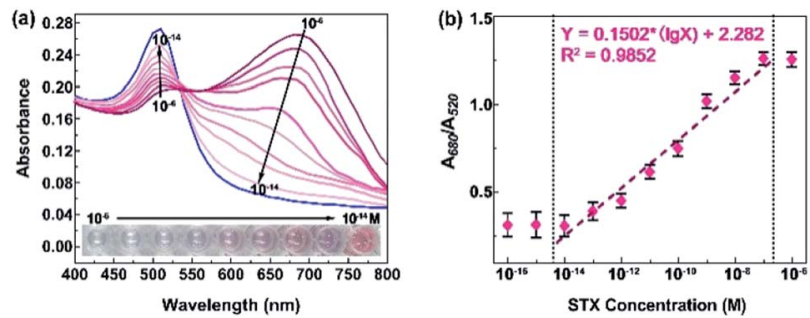

Fig. 4 Sensitivity of STX sensing. (a) Absorption spectrum and (b) $A_{680} /$ $A_{520}$ responses to different concentration of STX.

drop at $520 \mathrm{~nm}$ and color change from red to blue when the concentration increases from $0.2 \mathrm{M}$ to $0.4 \mathrm{M}$, which indicates the threshold lies in the range of $0.2-0.4 \mathrm{M}$. The detailed absorption spectra of $\mathrm{NaCl}$ concentration with fine steps shows the concentration threshold of $0.36 \mathrm{M}$. As a result, we use $0.36 \mathrm{M}$ $\mathrm{NaCl}$ to aggregate $\mathrm{Au}$ NPs in the subsequent detection.

Actually, $\mathrm{NaCl}$ concentration threshold is dependent on $\mathrm{Au}$ NPs density in the reaction system. A higher Au NPs density needs higher $\mathrm{NaCl}$ concentration threshold, as shown in Fig. $3 \mathrm{~b}$, and $\mathrm{NaCl}$ concentration threshold presents a linear relationship with Au NPs concentration. In our reaction system, the concentration of Au NPs is $47.8 \mu \mathrm{g} \mathrm{mL}^{-1}$ and $0.36 \mathrm{M} \mathrm{NaCl}$ is used to aggregate Au NPs.

During the sensing process, well-dispersed Au NPs aggregate after the addition of $\mathrm{NaCl}$, and return to the dispersion status after bonding with aptamers, because aptamers protect the charge balance of Au NPs from aggregation. ${ }^{32,33}$ The aptamer immobilization efficiency depends on incubation time with $\mathrm{Au}$ NPs. As shown in Fig. 3c, the color gradually changes from bluepurple back to red and the specific peaks recover from $680 \mathrm{~nm}$ to $520 \mathrm{~nm}$ with increasing incubation time, indicating that $\mathrm{Au}$ NPs get back to dispersed status after the bonding of aptamers. When the incubation time is more than 14 hours, the bonding of aptamers reaches saturation. Therefore, we chose 14 hours to immobilize aptamers with Au NPs.

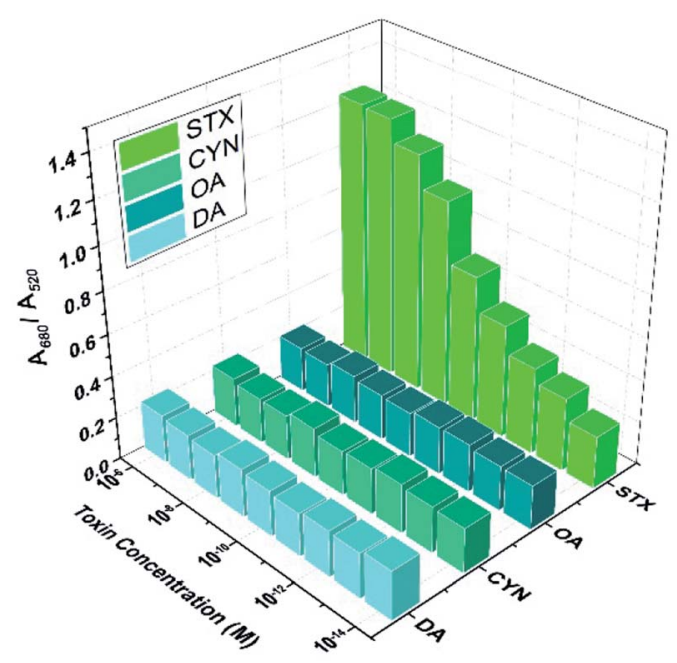

Fig. $5 A_{680} / A_{520}$ ratio of STX and other toxins with different concentrations. 
Table 2 Comparison of STX detection with different approaches ${ }^{a}$

\begin{tabular}{|c|c|c|c|c|c|c|}
\hline Method & Detector & Detection limit & Preparation time & Detection time & Detection cost & References \\
\hline MBA & Mouse & $250 \mathrm{pg}$ & $>1$ hour & 4-6 hours & High & 34 and 35 \\
\hline HPLC & No need & $0-27.6 \mathrm{nmol} \mathrm{g}^{-1}$ & $>20$ hours & $>2$ hours & High & 8 and 12 \\
\hline Immuno-chromatography & Antibody & $5.2 \mathrm{ng} \mathrm{mL}{ }^{-1}$ & $>10$ hours & $>1$ hour & High & 37 \\
\hline Immuno-assay & Antibody & $0.8-66.6 \mathrm{ng} \mathrm{mL}^{-1}$ & $>16$ hours & $36 \min$ & High & 38 \\
\hline ELISA & Antibody & $0.41-1.9 \mathrm{ng} \mathrm{g}^{-1}$ & $>8$ hours & $>100 \min$ & High & 10 and 39 \\
\hline Colorimetric & Aptamer & $10 \mathrm{fM}\left(3 \mathrm{fg} \mathrm{mL}^{-1}\right)$ & 14.5 hours & $30 \mathrm{~min}$ & Low & This work \\
\hline
\end{tabular}

${ }^{a}$ MBA is short for mouse bioassay. HPLC is short for high performance liquid chromatography. LC-MS is short for liquid chromatography-tandem mass spectrometry. ELISA is short for enzyme-linked immunosorbent assay.

STX aptamers bonding to Au NPs prevent them from aggregation even in the presence of $\mathrm{NaCl}$. After sample loading, STX is specifically bonded with STX aptamer, and Au NPs lose the protection from aptamers and aggregate with the assistance of $\mathrm{NaCl}$. Experiments show that STX reacts with aptamer in 10 minutes, and absorbance decreases at $520 \mathrm{~nm}$ while increases at $680 \mathrm{~nm}$ obviously, as in Fig. 3d. The solution color changes from red to blue-purple. Longer reaction time resulted to more adequate combination of STX aptamer and STX, and the reaction reaches saturation when it is more than $30 \mathrm{~min}$, and the absorbance curve gets stable. As a result, $30 \mathrm{~min}$ is selected to permit the sufficient specific capture of STX by aptamer.

\subsection{Detection of STX in seawater}

Sensitivity is one of the most parameter to evaluate biosensor and quantify the toxin concentration in test samples. In order to achieve the sensitivity, STX is mixed with seawater to form a series concentration from $0.1 \mathrm{fM}$ to $1 \mu \mathrm{M}$, and their absorbance spectra are tested, as shown in Fig. 4a. As the STX concentration increases, $A_{520}$ peak drops while $A_{680}$ ascends, and solution color changes from red to blue-purple. The ratio $A_{680} / A_{520}$ is applied to quantify toxin concentration, and Fig. $4 \mathrm{~b}$ shows its dependence on toxin concentration. In the range of 10 fM to $0.1 \mu \mathrm{M}, A_{680} / A_{520}$ ratio presents a linear relationship with STX concentration. The lowest STX detection concentration of $10 \mathrm{fM}$ is achieved, and STX concentration higher than $0.1 \mu \mathrm{M}$ is limited. However, higher concentration of STX sensing can be achieved by expanding reaction system, since it's easier to get saturated in smaller reaction system.

To evaluate the specificity of STX detection by aptamer and $\mathrm{Au}$ NPs based biosensor, STX and other three kinds of toxin are mixed with seawater, for instance, cylindrospermopsin (CYN), okdaic acid (OA) and domoic acid (DA). Experiments indicate that STX aptamer is specific only for STX toxin, and the $A_{680} /$ $A_{520}$ ratio of other three toxins is similar even their concentrations vary from $10^{-14} \mathrm{M}$ to $10^{-6} \mathrm{M}$, while it increases obviously with ascending STX concentration, as shown in Fig. 5. These results demonstrate the capability of aptamer and Au NPs based biosensor to conduct specific and sensitive STX detection.
Our biosensor is constructed by specific aptamer and chemically-synthesized Au NPs, which realizes ultrasensitive, fast, and economic detection of STX. In comparison with other approaches, as listed in Table 2, our biosensor achieves the lowest sensing concentration of $10 \mathrm{fM}\left(3 \mathrm{fg} \mathrm{mL}^{-1}\right)$ in detection time of 30 minutes. In addition, aptamer-Au NPs based biosensor is economic because it does not require expensive regents, complex process, and equipment during the whole process, which makes it promising in practical applications to monitor environmental pollution condition, marine aquaculture pollution, and seafood safety.

The detection sensitivity is strongly dependent on the sensing systems, including the binding affinity of STX, physical size of Au NPs, appropriate concentration match between $\mathrm{Au}$ NPs, STX aptamer and NaCl. The STX aptamer used in this work has much higher binding affinity than antibody. ${ }^{42,43}$ In the range of 5-20 nm, the peak displacement of Au NPs shows a linear relationship with increasing particle size. ${ }^{44}$ The average diameter of our Au NPs is $14.51 \mathrm{~nm}$, which is located in the most significant range of peak changes. Finally, the optimized concentrations of reaction regents in the whole system also plays important role in performance improvement.

\section{Conclusions}

In summary, we demonstrate an ultrasensitive, specific, fast, and economic colorimetric biosensor for STX detection. After optimization of reaction system, aptamer-Au NPs based biosensor achieves the lowest STX detection concentration of 10 $\mathrm{fM}\left(3 \mathrm{fg} \mathrm{mL} \mathrm{m}^{-1}\right)$ and sensing time of 30 minutes, presenting a linear relationship in the range of $10 \mathrm{fM}$ to $0.1 \mu \mathrm{M}$ aptamer-Au NPs based biosensor is a universal sensing platform, and it is applicable to other toxins detection. Further work is desired to realize a high throughput chip-based platform for multiplex toxins detection. The simple manipulation and economic expense empower the colorimetric biosensor promising applications in monitor environmental pollution condition, marine aquaculture pollution, and seafood safety.

\section{Conflicts of interest}

All the authors declare no conflicts of interest with this work. 


\section{Acknowledgements}

This work was supported by the National Key R\&D Plan of China (Grant No. 2019YFC1407800), the Major Innovation Projects in Shandong Province (2018YFJH0503), Natural Science Foundation for Distinguished Young Scientist of Shandong Province (Grant No. JQ201814), Natural Science Foundation for Young Scientists of China (Grant No. 61701282), Qilu Young Scholar Funds (11500086963060), the Fundamental Research Funds of Shandong University (2017TB0020 and 2017JC020), the National Natural Science Foundation of China (41876134) and Collaborative Innovation Center of Technology and Equipment for Biological Diagnosis and Therapy in Universities of Shandong. We would like to thank Xiaoju Li from State Key Laboratory of Microbial Technology, Shandong University for the assistance in HRTEM observation.

\section{References}

1 E. J. Schantz, et al., Paralytic shellfish poison. VI. A procedure for the isolation and purification of the poison from toxic clam and mussel tissues, J. Am. Chem. Soc., 1957, 79, 52305235.

2 M. Wiese, P. M. Dagostino, T. K. Mihali, M. C. Moffitt and B. A. Neilan, Neurotoxic alkaloids: saxitoxin and its analogs, Mar. Drugs, 2010, 8, 2185-2211.

3 I. Falconer and A. Humpage, Health risk assessment of cyanobacterial (blue-green algal) toxins in drinking water, Int. J. Environ. Res. Public Health, 2005, 2, 43-50.

4 S. Faber, Saxitoxin and the induction of paralytic shellfish poisoning, J. Young Investig., 2012, 23, 1-7.

5 A. Capper, L. J. Flewelling and K. Arthur, Dietary exposure to harmful algal bloom (HAB) toxins in the endangered manatee (Trichechus manatus latirostris) and green sea turtle (Chelonia mydas) in Florida, USA, Harmful Algae, 2013, 28, 1-9.

6 G. Isbister, Textbook of Adult Emergency Medicine, Churchill Livingstone, 2001.

7 I. Chorus, Current approaches to cyanotoxin risk assessment, risk management and regulations in different countries, Fed. Environmental Agency, 2005.

8 Y. Liu, et al., Paralytic shellfish toxins in phytoplankton and shellfish samples collected from the Bohai Sea, China, Mar. Pollut. Bull., 2017, 115, 324-331.

9 S. Tsuchiya, et al., Synthesis and identification of proposed biosynthetic intermediates of saxitoxin in the cyanobacterium Anabaena circinalis (TA04) and the dinoflagellate Alexandrium tamarense (Axat-2), Org. Biomol. Chem., 2014, 12, 3016-3020.

10 J. R. McCall, W. C. Holland, D. M. Keeler, D. R. Hardison and R. W. Litaker, Improved Accuracy of Saxitoxin Measurement Using an Optimized Enzyme-Linked Immunosorbent Assay, Toxins, 2019, 11, 632.

11 J. Regueiro, A. E. Rossignoli, G. álvarez and J. Blanco, Automated on-line solid-phase extraction coupled to liquid chromatography-tandem mass spectrometry for determination of lipophilic marine toxins in shellfish, Food Chem., 2011, 129, 533-540.

12 R. Watanabe, et al., Development of Ultra-Performance Liquid Chromatography with Post-Column Fluorescent Derivatization for the Rapid Detection of Saxitoxin Analogues and Analysis of Bivalve Monitoring Samples, Toxins, 2019, 11, 573.

13 K. Petropoulos, S. F. Bodini, et al., Re-modeling ELISA kits embedded in an automated system suitable for on-line detection of algal toxins in seawater, Sens. Actuators, B, 2019, 283, 865-872.

14 W. Zhao, M. M. Ali, S. D. Aguirre, M. A. Brook and Y. Li, Paper based bioassays using gold nanoparticle colorimetric probes, Anal. Chem., 2008, 80, 8431-8437.

15 L. Guo, G. Chen and D. H. Kim, Three-Dimensionally Assembled Gold Nanostructures for Plasmonic Biosensors, Anal. Chem., 2010, 82, 5147-5153.

$16 \mathrm{~L}$. Wei, et al., Colorimetric assay for protein detection based on "nano-pumpkin" induced aggregation of peptidedecorated gold nanoparticles, Biosens. Bioelectron., 2015, 71, 348-352.

17 Y. Leng, et al., Gold-nanoparticle-based colorimetric array for detection of dopamine in urine and serum, Talanta, 2015, 139, 89-95.

18 Y. Leng, K. Jiang, W. Zhang and Y. Wang, Synthesis of gold nanoparticles from $\mathrm{Au}$ (I) ions that shuttle to solidify: application on the sensor array design, Langmuir, 2017, 33, 6398-6403.

19 Z.-J. Xie, X.-Y. Bao and C.-F. Peng, Highly Sensitive and Selective Colorimetric Detection of Methylmercury Based on DNA Functionalized Gold Nanoparticles, Sensors, 2018, 18, 2679.

$20 \mathrm{~J}$. Li, C. Kong, Q. Liu and Z. Chen, Colorimetric ultrasensitive detection of DNA based on the intensity of gold nanoparticles with dark-field microscopy, Analyst, 2018, 143, 4051-4056.

21 Y. Jiao, Q. Liu, H. Qiang and Z. Chen, Colorimetric detection of L-histidine based on the target-triggered self-cleavage of swing-structured DNA duplex-induced aggregation of gold nanoparticles, Microchim. Acta, 2018, 185, 452.

22 E. Priyadarshini and N. Pradhan, Metal-induced aggregation of valine capped gold nanoparticles: an efficient and rapid approach for colorimetric detection of $\mathrm{Pb}^{2+}$ ions, Sci. Rep., 2017, 7, 9278.

$23 \mathrm{~J}$. Qiu, et al., Colorimetric detection of $\mathrm{Ba}^{2+}, \mathrm{Cd}^{2+}$ and $\mathrm{Pb}^{2+}$ based on a multifunctionalized Au NP sensor, Analyst, 2019, 144, 5081-5089.

$24 \mathrm{Y}$. Zhang, et al., A flower-like $\mathrm{MoS}_{2}$-decorated $\mathrm{MgFe}_{2} \mathrm{O}_{4}$ nanocomposite: mimicking peroxidase and colorimetric detection of $\mathrm{H}_{2} \mathrm{O}_{2}$ and glucose, Sens. Actuators, B, 2018, 275, 155-162.

25 Y. Ding, et al., FePt-Au ternary metallic nanoparticles with the enhanced peroxidase-like activity for ultrafast colorimetric detection of $\mathrm{H}_{2} \mathrm{O}_{2}$, Sens. Actuators, B, 2018, 259, 775-783.

$26 \mathrm{Y}$. Zhou and Z. Ma, Colorimetric detection of $\mathrm{Hg}^{2+}$ by $\mathrm{Au}$ nanoparticles formed by $\mathrm{H}_{2} \mathrm{O}_{2}$ reduction of $\mathrm{HAuCl}_{4}$ using 
Au nanoclusters as the catalyst, Sens. Actuators, B, 2017, 241, 1063-1068.

27 Y. Leng, et al., Protein-directed synthesis of highly monodispersed, spherical gold nanoparticles and their applications in multidimensional sensing, Sci. Rep., 2016, 6, 28900.

28 Y. Yang, et al., Controlled surface-plasmon coupling in $\mathrm{SiO}_{2}$ coated gold nanochains for tunable nonlinear optical properties, Appl. Phys. Lett., 2006, 88, 081110.

29 Y. Yang, S. Matsubara, M. Nogami and J. Shi, Controlling the aggregation behavior of gold nanoparticles, Mater. Sci. Eng., 2007, 140, 172-176.

$30 \mathrm{X}$. Zheng, et al., A saxitoxin-binding aptamer with higher affinity and inhibitory activity optimized by rational sitedirected mutagenesis and truncation, Toxicon, 2015, 101, 41-47.

31 R. R. Guillard and J. H. Ryther, Studies of marine planktonic diatoms: I. Cyclotella nana Hustedt, and Detonula confervacea (Cleve) Gran, Can. J. Microbiol., 1962, 8, 229-239.

$32 \mathrm{Y}$. Xu, et al., Colorimetric detection of kanamycin based on analyte-protected silver nanoparticles and aptamerselective sensing mechanism, Anal. Chim. Acta, 2015, 891, 298-303.

33 H. Wei, B. Li, J. Li, E. Wang and S. Dong, Simple and sensitive aptamer-based colorimetric sensing of protein using unmodified gold nanoparticle probes, Chem. Commun., 2007, 3735-3737.

34 R. Manger, et al., Detection of sodium channel toxins: directed cytotoxicity assays of purified ciguatoxins, brevetoxins, saxitoxins, and seafood extracts, J. AOAC Int., 1995, 78, 521-527.

35 T. Takagi, K. Hayashi and Y. Itabashi, Toxic effect of free unsaturated fatty acids in the mouse assay of diarrhetic shellfish toxin [separated from the toxic scallops
Patinopecten yessoensis] by intraperitoneal injection, Bull. Jpn. Soc. Sci. Fish., 1984, 50(8), 1413-1418.

36 D. T. Harwood, et al., Paralytic shellfish toxins, including deoxydecarbamoyl-STX, in wild-caught Tasmanian abalone (Haliotis rubra), Toxicon, 2014, 90, 213-225.

37 K. Su, X. Qiu, J. Fang, Q. Zou and P. Wang, An improved efficient biochemical detection method to marine toxins with a smartphone-based portable system-Bionice-Eye, Sens. Actuators, B, 2017, 238, 1165-1172.

$38 \mathrm{X}$. Zhang and Z. Zhang, Capillary electrophoresis-based immunoassay with electrochemical detection as rapid method for determination of saxitoxin and decarbamoylsaxitoxin in shellfish samples, J. Food Compos. Anal., 2012, 28, 61-68.

39 S. E. Fire, J. A. Browning, W. N. Durden and M. K. Stolen, Comparison of during-bloom and inter-bloom brevetoxin and saxitoxin concentrations in Indian River Lagoon bottlenose dolphins, 2002-2011, Aquat. Toxicol., 2019, 105371.

40 L. Zhong, et al., Portable Smartphone-based Colorimetric Analyzer with Enhanced Gold Nanoparticles for On-site Tests of Seafood Safety, Anal. Sci., 2019, 35, 133-140.

41 B. S. Cheun, M. Loughran, T. Hayashi, Y. Nagashima and E. Watanabe, Use of a channel biosensor for the assay of paralytic shellfish toxins, Toxicon, 1998, 36, 1371-1381.

$42 \mathrm{~S}$. Basu, et al., Biomolecule induced nanoparticle aggregation: effect of particle size on interparticle coupling, J. Colloid Interface Sci., 2007, 313, 724-734.

$43 \mathrm{H}$. Xu, et al., Aptamer-Functionalized Gold Nanoparticles as Probes in a Dry-Reagent Strip Biosensor for Protein Analysis, Anal. Chem., 2009, 81, 669-675.

$44 \mathrm{~J}$. Guo, et al., Aptamer-based fluorescent screening assay for acetamiprid via inner filter effect of gold nanoparticles on the fluorescence of CdTe quantum dots, Anal. Bioanal. Chem., 2016, 408, 557-566. 